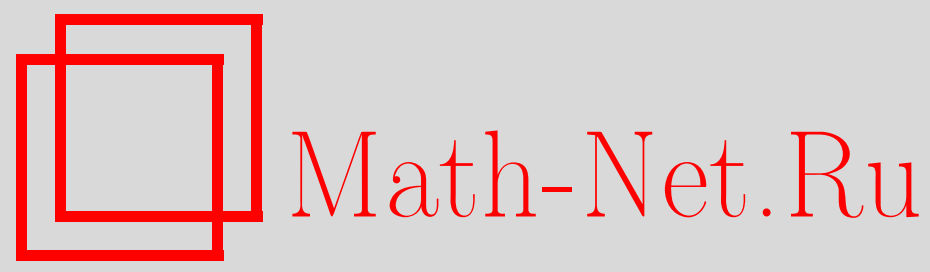

В. П. Маслов, Фазовые переходы нулевого рода, Матем. заметки, 2004, том 76, выпуск 5, 748-761

DOI: https://doi.org/10.4213/mzm145

Использование Общероссийского математического портала Math-Net.Ru подразумевает, что вы прочитали и согласны с пользовательским соглашением http://www . mathnet.ru/rus/agreement

Параметры загрузки:

IP : 34.229 .45 .116

26 апреля 2023 г., 13:08:26

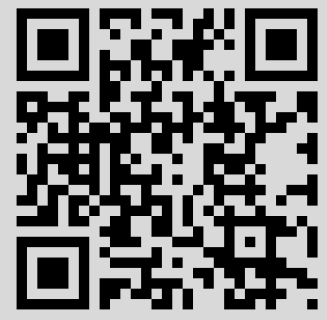


УДК 517.983

\title{
ФАЗОВЫЕ ПЕРЕХОДЫ НУЛЕВОГО РОДА
}

\author{
В.П. Маслов
}

\begin{abstract}
В теории сверхтекучести и сверхпроводимости теоретически обнаружен скачок свободной энергии, который естественно назьвать фазовым переходом нулевого рода. Приводится пример точно решаемой задачи, в которой этот фазовый переход имеет место.

Библиографоия: 24 названия.
\end{abstract}

\section{1. Общие соображения о фазовом переходе нулевого рода}

1.1. Основные большие параметры термодинамики. Термодинамика изучает установившиеся процессы, когда независимо от своего начального состояния система приходит в состояние, котороев дальнейшем ужене меняется. Если жевсе-таки такое состояние меняется, то изменение будет термодинамическим только в том случае, если процесс совершается чрезвычайно медленно [1]. Иначе говоря, мы бесконечно мало меняем состояние системы и ждем пока она не придет в равновесие и не будет зависеть от начального состояния.

Таким образом, здесь незримо присутствует большой параметр - время, т.е. мы рассматриваем систему через огромные промежутки времени. С другой стороны, термодинамика является предельной макроскопической теорией, получающейся из микроскопической статистической физики при числе частиц $N \rightarrow \infty$. Следовательно, имеется два больших параметра и от их соотношения многое зависит. В свою очередь классическая статистическая физика является пределом квантовой статистики при $h \rightarrow 0$ ( $h$ - параметр Планка). Таким образом, “сталкиваются” уже три больших параметра: масштаб времени, число частиц и $1 / h$.

Термодинамика рассматривает часто и достаточно большие периоды времени, в которые лиш часть системы приходит в равновесие, так называемое “локальное равновесие". Например, при нагревании плазмы магнитньм полем через некоторое большое наблюдаемое время ионы распределяются по Максвеллу, и лишь через сушественно большее время вся система приходит в термодинамическое равновесие.

1.2. Постулат Гиббса. Имеет место постулат Гиббса, которьй в квантовой статистике звучит следующим образом.

Пусть система характеризуется оператором Гамильтона $\widehat{H}$ в гильбертовом пространстве (в частности, в пространстве Фока) $\Phi$. Пусть оператор $\widehat{H}$ имеет неотрицательньй 
дискретный спектр $\lambda_{0}, \lambda_{1}, \ldots, \lambda_{n} \ldots$ Тогда свободная энергия, зависящая от температуры $\theta$, определяется как

$$
E=\theta \ln \sum_{n=0}^{\infty} e^{-\lambda_{n} / \theta} \delta_{n}
$$

где $\delta_{n}-$ кратность собственного значения $\lambda_{n}$.

Отметим, что в постулате Гиббса появляется еще один параметр, а именно

$$
E=\theta \ln \lim _{M \rightarrow \infty} \sum_{n=0}^{M} e^{-\lambda_{n} / \theta} \delta_{n}
$$

Это один из важнейших моментов, так как $\lim _{M \rightarrow \infty}$ и $\lim _{N \rightarrow \infty}$ не коммутируют! Оказьвается, сначала нужно перейти при $M \rightarrow \infty$, а затем при $N \rightarrow \infty$.

Когда речь идет о локальном равновесии, то в формуле Гиббса, как правило, рассматривается некоторое подмножество $\left\{\lambda_{n^{\prime}}\right\}$ множества $\left\{\lambda_{n}\right\}$. Это нужно делать, исследуя вышеупомянутую задачу о распределении ионов в плазме при нагревании. Это же делается в стекле и ряде других физических задач.

1.3. Оператор “трения". При температуре $\theta=0$ формула Гиббса переходит в значение нижнего собственного значения, которое в физике называется "основным состоянием".

Суть заключается в том не сформулированном четко законе, который Боголюбов и другие назьвают “энергетической выгодностью”. Энергетически выгодна болеенизкая энергия. Например, если мы возмущаем систему некоторым оператором $\widehat{V}:(\Phi \rightarrow \Phi)$, то если он достаточно мал, то матрица перехода из состояния $\lambda_{n}$ в состояние $\lambda_{m}$ определяется матричным элементом $\left(\Psi_{n} \widehat{V} \Psi_{m}^{*}\right)$, где $\Psi_{n}$ и $\Psi_{m}$ - собственные функции, отвечающие $\lambda_{n}$ и $\lambda_{m}$ соответственно (см. [2]).

Квадрат этого матричного элемента есть вероятность перехода из состояния с энергией $\lambda_{n}$ в состояние с энергией $\lambda_{m}$. Но если $\lambda_{n}<\lambda_{m}$, то этот переход "энергетически невьгоден" и поэтому как бы с точки зрения статфизики и термодинамики неосуществим, т.е. с математической точки зрения его надо положить равным нулю. Тогда матрица перехода будет несамосопряженной с нулями вьше диагонали. Это означает учет трения. Маятник колеблется и при учете трения в конще концов остановится, т.е. попадет в “основное состояние". Более подробно оператор “трения" рассмотрен в работе автора [3].

1.4. Фазовые переходы. Производная $\varepsilon(\theta)$ по $\theta$ назьвается энтропией, и когда в некоторой точке $\theta_{0}$ она испытывает скачок, то говорят, что осуществляется фазовьй переход первого рода; когда ее вторая производная испытывает скачок, то - фазовьй переход второго рода и т.д.

Но разумеется, не сама функция испытывает скачок, а ее главньй член асимптотики при $N \rightarrow \infty$. Этот “скачок" может быть иногда реально на эксперименте растянут по времени, но мы условились рассматривать изменения на достаточно больших промежутках времени. В этих промежутках могут происходить не термодинамические, а динамические процессы. В термодинамике мы ими пренебрегаем, рассматривая только времена, когда система пришла в равновесие с учетом “энергетической вьгодности” [4]. 
Фазовьй переход нулевого рода автор обнаружил как в теории сверхтекучести и сверхпроводимости, так и в экономике (пробой курса акций, дефолт), которая неожиданно для самого автора оказалась в силу естественной аксиоматики (см. [5]) связана с квантовой статистикой и термодинамикой. Этот фазовьй переход не был замечен физиками и, казалось бы, противоречит их представлениям о том, что свободная энергия определяется с точностью до константы.

1.5. Метастабильное состояние. Мы рассмотрим простой пример квазиклассического приближения одномерного уравнения Шрёдингера

$$
-h^{2} y_{n}^{\prime \prime}+u(x) y_{n}=\lambda_{n} y_{n}, \quad y_{n}(x) \in L_{2}, \quad h \ll 1
$$

где $u(x)=\left(x^{2}-1\right)^{2}+q x, q>0-$ константа.

В классической механике - это как бы две чашки, соединенные между собой барьером. Если частица находится на дне правой более высокой чашки, то она не перекатится во вторую, более низкую, если только барьер не исчезнет.

$\mathrm{C}$ точки зрения постулата Гиббса при $\theta \rightarrow 0$ частица должна оказаться в более глубокой впадине. Вместе с тем очевидно, что если $h \ll 1$, то в высокой впадине она будет находиться очень длительное время. В формуле Гиббса в этом случае нужно проводить суммирование по подмножеству собственньх значений, отвечаюших болеевысокой впадине, собственные функции которых стремятся к нулю при $h \rightarrow 0$ вне этой впадины. При этом температура $\theta$ должна быть не слишком велика, чтобы собственные значения вьше барьера не играли бы роль в формуле Гиббса.

Такое состояние в локальном минимуме потенциальной ямы $u(x)$ есть пример метастабильного состояния.

Если мы рассматриваем элемент матрицы перехода с нижнего уровня, отвечающего высокой впадине, на самый нижний уровень $\lambda_{0}$ (на дне глубокой впадины), то он оказывается экспоненциально мал по $h$, переход же на высокие уровни запрещен в силу закона “энергетической вьгодности" (т.е. мы рассматриваем возмущение “трения").

1.6. Сверхтекучесть. Как показал Боголюбов, использовавший некоторые идеи Ландау (см. сноску на с. 219 в [6]), сверхтекучесть не есть движение жидкости, не есть динамика, а есть состояние - как, например, лед и пар для воды.

Оно отвечает некоторому метастабильному состоянию системы, переход из которого в нормальное почти запрещен: он экспоненциально мал при $N \rightarrow \infty$.

Боголюбов доказал это строго при условии периодичности для системы уравнений Шрёдингера, иначе говоря, на торе. Спектр сверхтекучих скоростей (уровней энергии, отвечающих соответствующим импульсам) был дискретный. Это сразу отличает состояние сверхтекучести от гидродинамики жидкости. В пределе, когда радиус тора устремлялся к бесконечности, спектр отнюдь не переходил в непрерьвньй, как это обычно бывает, а становился всюду плотным, точечным.

В свое время автор впал в такую ошибку для одной задачи одномерного уравнения Шрёдингера, считая, что спектр непрерьвный, в то время как он оказался всюду плотным точечным. Это касалось контрпримера, приведенного мною в 1960 году к теореме Костюченко-Морена-Гельфанда-Шилова. Все они и все специалисты признали этот контрпример, нашли у себя ошибки, и только через 20 лет Харрис нашел "дыру" в моем доказательстве, о чем сообщил Бари Саймон в своей обширной работе. После этого 
С. Молчанов и я обнаружили, что пример ошибочный и спектр не непрерывный, что казалось всем очевидным, а всюду плотный точечньй. И лишь в 1987 году нам с Молчановым удалось, используя ту же идею, найти контрпример с чисто непрерьвным спектром [7].

С физической точки зрения, на мой взгляд, тот факт, что спектр является в пределе всюду плотным точечньм, легко объясним.

Действительно, если система находится в состоянии со сверхтекучей скоростью $v$, то переход ее в большую скорость запрещен законом “выгодности энергетического состояния", а в сколь угодно меньшую - тем, что уменьшение скорости противоречит понятию сверхтекучести. Что же касается математической точки зрения, то чем радиус тора больше, тем переход из одного состояния в другое меньше (экспоненциально меньше).

1.7. Фазовый переход нулевого рода. 1.7.1. Автором был объяснен эффект фонтанирования, открытый в 1938 году Дж. Алленом и Х. Джонсом, когда по капилляру диаметром $10^{-4}$ см “текла" сверхтекучая жидкость (на самом деле находилась на сверхтекучем уровне метастабильного состояния).

На двухуровневой модели автором было показано, что в точке, нагретой (светом) до температуры фазового перехода $\theta_{c}$ происходит обращение в бесконечность теплоемкости, скачок энтропии и свободной энергии до нижнего значения свободной энергии, т.е. до той точки, в которую приходит кривая из основного состояния, нагретого до температуры $\theta_{c}$. Это значит, что происходит фазовый переход нулевого рода. Мы покажем в настоящей статье, что та же картина возникает и в модели Боголюбова слабо-неидеального бозе-газа [8].

Это явление легко объясняется, если предположить, что сверхтекучесть не термодинамическое состояние, а движение жидкости. Жидкость становится при $\theta=\theta_{c}$ вязкой и не может проникать в тонкий капилляр.

Но дело в том, что это не движение, а состояние, и тогда это - переходнулевого рода.

Возникает вопрос, что будет происходить со сверхтекучестью не в капилляре, а достаточно толстой трубе. Где фазовьй переход нулевого рода? При $\theta=\theta_{c}$ жидкость из сверхтекучего состояния переходит в обычную жидкость с вязкостью и начинает течь по общим законам гидродинамики. Через некоторое время она остановится, а переход из сверхтекучего состояния в состояние покоящейся жидкости есть термодинамический переход. Все промежуточное течение - гидродинамика, которая не должна учитываться в нашем масштабе времени.

1.7.2. Рассмотрим подробнее пример 1.6. Будем медленно менять константу $q$. Отметим, что в термодинамике, учитывающей поле, имеются еще две термодинамические величины: напряженность поля и заряд. Таким образом, изменение $q$ есть изменение термодинамической переменной.

Покажем, что переход $q$ через точку нуль приводит к фазовому переходу нулевого рода. Действительно, при $q=0$ возникает резонанс: собственные функции уже не сосредоточены в одной из впадин и вероятность (квадрат каждой собственной функции) одинаково распределена по обоим впадинам. Собственных значений становится "вдвое" больше и распределение Гиббса меняется скачком. Именно здесь можно увидеть роль времени. Чтобы половина функции, убывающей внепервой ямы, “перекачалась" во вторую, должно пройти большое время. Однако формула Гиббса это не учитывает. Ждать этого перехода, может быть, придется долго, как и в предыдущем примере с толстой трубой. 
Если $q$ становится отрицательным, то остаются все собственные функции как в одной, так и в другой впадине, и формула Гиббса берется по всем собственным значениям, а не по их подмножеству.

1.7.3. Наконец, остановимся на эффекте перехода в турбулентное течение для жидкого гелия, которьй по существу очень близок к идее Ландау о перекачке энергии между крупными и мелкими вихрями.

Как мы покажем в другой работе, резонанс между этими двумя типами вихрей и приводит к фазовому переходу нулевого рода, который резко меняет термодинамические параметры с точки зрения термодинамики, т.е. учитьвая большие масштабы времени между переходами, и, естественно, осреднение по этим временам.

1.7.4. Поскольку для денежных знаков несущественны их номера, а играет роль только их величина, и обмен двух купюр одного достоинства не есть операция, то они подчиняются статистике Бозе. Осреднение доходов есть нелинейная операция, как и сложение. Оказалось, что единственная нелинейная “арифметика" (полукольцо), которая удовлетворяет естественньм аксиомам осреднения для бозе-частиц - купюр, имеет вид $a \oplus b=\ln \left(e^{a}+e^{b}\right)$.

Это приводит к формуле типа Гиббса. Изменение $\beta=1 / T$ может трактоваться как изменение покупательной способности рубля, например, печатание новых купюр. После этого в течение некоторого времени снова устанавливается баланс (равновесие).

Роль энергетической выгодности играет обычная финансовая выгодность. Фазовые переходы нулевого рода имеют смысл дефолта или кризиса [5], [9].

\section{2. Точно решаемая модель}

Вначале мы рассмотрим одномерное уравнение Шрёдингера для одной частицы на окружности

$$
\widehat{H} \psi_{k}(x)=E \psi_{k}(x), \quad \psi_{k}(x-L)=\psi_{k}(x),
$$

где $\psi_{k}(x)$ - волновая функция, $x$ принимает значения на окружности, а $\widehat{H}$ - дифференциальньй оператор вида

$$
\widehat{H}=\varepsilon(-i \hbar \partial / \partial x), \quad \varepsilon(z) \in C^{\infty}
$$

здесь $\hbar-$ постоянная Планка, $\psi_{k}(x)=\exp \left(i p_{k} x\right)$, где $p_{k}=2 \pi \hbar k / L, k-$ произвольное целое число, соответствуюшие собственные значения равны

$$
E_{k}=\varepsilon\left(p_{k}\right)
$$

Перейдем от гамильтоновой функции $\varepsilon(p)$ к дискретной функции $\widetilde{\varepsilon}(p)$, имеющей вид

$$
\widetilde{\varepsilon}(p)=\varepsilon(n \Delta p) \quad \text { при } \quad \Delta p\left(n-\frac{1}{2}\right) \leqslant p<\Delta p\left(n+\frac{1}{2}\right),
$$

где $n$ - произвольное целое число, $\Delta p$ - некоторая положительная постоянная. Гамильтониан (2) при таком переходе изменяется соответствующим образом, будем обозначать его $\widehat{\widetilde{H}}$, собственные функции этого оператора совпадают с $\psi_{k}(x)$, а собственные значения (3) переходят в

$$
\widetilde{E}_{k}=\widetilde{\varepsilon}\left(p_{k}\right) \text {. }
$$


Будем далее считать, что постоянная $\Delta p$ имеет вид

$$
\Delta p=\frac{2 \pi \hbar G}{L}
$$

где $G$ - натуральное число. В таком случае из (4) следует, что набор энергетических уровней (5) является набором $G$-кратно вырожденных энергетических уровней

$$
\lambda_{n}=\widetilde{E}_{G n}=\varepsilon\left(p_{G n}\right) .
$$

Уравнение Шрёдингера для $N$ не взаимодействующих частиц имеет вид

$$
\widehat{H}_{N} \Psi\left(x_{1}, \ldots, x_{N}\right)=E \Psi\left(x_{1}, \ldots, x_{N}\right),
$$

где $\Psi\left(x_{1}, \ldots, x_{N}\right)$ - симметричная функция переменных $x_{1}, \ldots, x_{N}$ (бозоны), а гамильтониан $\widehat{H}_{N}$ выражается формулой

$$
\widehat{H}_{N}=\sum_{j=1}^{N} \widehat{\widetilde{H}}_{j}
$$

где $\widehat{\widetilde{H}}_{j}-$ гамильтониан частицы с номером $j$, который имеет вид

$$
\widehat{\widetilde{H}}_{j}=\widetilde{\varepsilon}\left(-i \hbar \frac{\partial}{\partial x_{j}}\right)
$$

Полная ортонормированная система симметричных собственных функций гамильтониана (9) имеет вид

$$
\Psi_{\{N\}}\left(x_{1}, \ldots, x_{N}\right)=\operatorname{Symm}_{x_{1}, \ldots, x_{N}} \psi_{\{N\}}\left(x_{1}, \ldots, x_{N}\right),
$$

где $\operatorname{Symm}_{x_{1}, \ldots, x_{N}}$ - оператор симметризации по переменным $x_{1}, \ldots, x_{N},\{N\}$ - набор целых неотрицательных чисел $N_{k}, k \in \mathbb{Z}$, удовлетворяющих условию

$$
\sum_{k=-\infty}^{\infty} N_{k}=N
$$

а функция $\psi_{\{N\}}\left(x_{1}, \ldots, x_{N}\right)$ равна

$$
\psi_{\{N\}}\left(x_{1}, \ldots, x_{N}\right)=\prod_{s=1}^{N} \psi_{k_{s}}\left(x_{s}\right)
$$

где индексы $k_{1}, \ldots, k_{N}$ выражаются через набор $\{N\}$ из условий

$$
k_{s} \leqslant k_{s+1} \quad \text { для всех } 1 \leqslant s \leqslant N-1, \quad \sum_{s=1}^{N} \delta_{k k_{s}}=N_{k} \quad \text { для всех } k \in \mathbb{Z}
$$


здесь $\delta_{k k^{\prime}}$ - символ Кронекера. Собственные значения гамильтониана (9) равны

$$
E(\{N\})=\sum_{k=-\infty}^{\infty} \widetilde{E}_{k} N_{k}
$$

Рассмотрим взаимодействие между частицами следующего вида. Будем считать, что частицы взаимодействуют парами и оператор взаимодействия частищ с номерами $j$ и $k$ имеет вид

$$
\widehat{V}_{j k}=-\frac{V}{N} W\left(\widehat{\widetilde{H}}_{j}-\widehat{\widetilde{H}}_{k}\right),
$$

где $V>0$ - параметр величины взаимодействия, а функция $W(\xi)$ равна

$$
W(\xi)= \begin{cases}1 & \text { при }|\xi|<D, \\ 0 & \text { при }|\xi| \geqslant D ;\end{cases}
$$

здесь $D>0$ - параметр ширины взаимодействия по энергии. Оператор (17) соответствует взаимодействию, при котором пара частиц притягивается друг к другу и испускает квант энергии $-V / N$, если разность их энергий меньше $D$, а если разность энергий частиц больше $D$, то частицы не взаимодействуют. Гамильтониан системы $N$ частиц со взаимодействием (17) принимает вид

$$
\widehat{H}_{N}=\sum_{j=1}^{N} \widehat{\widetilde{H}}_{j}+\sum_{j=1}^{N} \sum_{k=j+1}^{N} \widehat{V}_{j k}
$$

В силу (16) суммы в выражении (18) коммутируют, поэтому набор собственных функций гамильтониана (18) совпадает с (11), также из (16) следует, что соответствующие собственные значения равны

$$
\mathbf{E}(\{N\})=\sum_{k=-\infty}^{\infty} \widetilde{E}_{k} N_{k}-\frac{V}{2 N} \sum_{k=-\infty}^{\infty} \sum_{l=-\infty}^{\infty} W\left(\widetilde{E}_{k}-\widetilde{E}_{l}\right)\left(N_{k} N_{l}-\delta_{k l} N_{k}\right) .
$$

Далее будем считать, что ширина взаимодействия достаточно мала и для нее вьполняется условие

$$
D<\min _{n \neq m}\left|\lambda_{n}-\lambda_{m}\right|
$$

Набор энергетических уровней $\widetilde{E}_{k}, k \in \mathbb{Z}$, совпадает с $G$-кратно вырожденным набором уровней (7). Значит в силу (20), набор энергетических уровней (19) рассматриваемой системы $N$ частиц может быть записан в виде

$$
\mathbf{E}(\{\tilde{N}\})=\sum_{n=-\infty}^{\infty} \lambda_{n} \tilde{N}_{n}-\frac{V}{2 N} \sum_{n=-\infty}^{\infty} \tilde{N}_{n}\left(\tilde{N}_{n}-1\right)
$$

где уровень $\mathbf{E}\{\tilde{N}\})$ имеет кратность

$$
\Gamma(\{\tilde{N}\})=\prod_{n=-\infty}^{\infty} \frac{\left(G+\widetilde{N}_{n}-1\right) !}{(G-1) ! \widetilde{N}_{n} !}
$$


Здесь $\{\tilde{N}\}$ обозначает набор целых неотрицательных чисел $\widetilde{N}_{n}, n \in \mathbb{Z}$, для которых выполняется условие

$$
\sum_{n=-\infty}^{\infty} \tilde{N}_{n}=N
$$

Рассмотрим статистическую сумму для системы $N$ бозонов с гамильтонианом (18). Так как энергетические уровни и их кратности выражаются формулами (21) и (22) соответственно, то статистическая сумма при температуре $\theta$ принимает вид

$$
Z(\theta, N)=\sum_{\{\tilde{N}\}} \Gamma(\{\tilde{N}\}) \exp ((-\mathbf{E}\{\tilde{N}\}) \theta)
$$

суммирование здесь берется по всем наборам $\{\tilde{N}\}$ с учетом условия (23).

Будем считать дальше, что $G$ зависит от $N$, причем вьполняется условие

$$
\lim _{N \rightarrow \infty} \frac{G}{N}=g>0
$$

Обозначим через $\widetilde{F}(\{\tilde{N}\}, \theta)$ функцию вида

$$
\widetilde{F}(\{\tilde{N}\}, \theta)=\mathbf{E}(\{\tilde{N}\})-\theta \ln (\Gamma(\{\tilde{N}\})) ;
$$

$\left\{\tilde{N}^{0}\right\}$ - набор неотрицательных чисел $\tilde{N}_{n}^{0}, n \in \mathbb{Z}$, для которых функция $(26)$ минимальна при условии (23).

Рассмотрим теперь вопрос о нахождении минимального значения функции (26) при условии (23). В пределе при $N \rightarrow \infty$ и при выполненном условии (25) точка минимума имеет вид

$$
\tilde{N}_{n}(\theta)=N\left(m_{n}(\theta)+\mathrm{o}(1)\right)
$$

где $m_{n}(\theta), n \in \mathbb{Z}$, определяется из системы уравнений

$$
\lambda_{n}-V m_{n}+\theta \ln \left(\frac{m_{n}}{g+m_{n}}\right)=\mu(\theta), \quad n \in \mathbb{Z},
$$

а $\mu(\theta)$ находится из уравнения

$$
\sum_{n=-\infty}^{\infty} m_{n}=1
$$

Подстановка (27) в (26) и использование при этом асимптотической формулы Стирлинга приводит к следующему равенству для удельной свободной энергии:

$$
\begin{aligned}
f(\theta) & \equiv \lim _{N \rightarrow \infty} f(\theta, N)=\lim _{N \rightarrow \infty} \frac{\tilde{F}\left(\left\{\tilde{N}^{0}\right\}, \theta\right)}{N} \\
& =\sum_{n=-\infty}^{\infty}\left(\lambda_{n} m_{n}-\frac{V}{2} m_{n}^{2}\right)+\left(\theta m_{n} \ln \left(\frac{m_{n}}{g}\right)-\theta\left(g+m_{n}\right) \ln \left(1+\frac{m_{n}}{g}\right)\right),
\end{aligned}
$$

где для краткости написания формулы у $m_{n}(\theta)$ опущен аргумент $\theta$. Введем обозначение

$$
\omega_{n}=\lambda_{n}-V m_{n}
$$


ЗАмЕчАниЕ. Система уравнений (28) и (29) в обозначениях (31) принимает вид

$$
\begin{aligned}
\omega_{n}(\theta)= & \lambda_{n}-V \frac{g}{\exp \left(\left(\omega_{n}-\mu\right) / \theta\right)-1}, \quad n \in \mathbb{Z}, \\
& \sum_{n=-\infty}^{\infty} \frac{g}{\exp \left(\left(\omega_{n}-\mu\right) / \theta\right)-1}=1 .
\end{aligned}
$$

Система уравнений (32), (33) точно совпадает с температурными уравнениями Хартри [10] для системы $N$ бозонов с гамильтонианом (18).

Исследуем решения системы уравнений $(28),(29)$. При $\theta=0$ система имеет множество решений, которые будем нумеровать целым числом $l$ :

$$
m_{n}^{(l)}=\delta_{l n}, \quad n, l \in \mathbb{Z} .
$$

Отберем из всех номеров $l$ такие, для которых вьполняется условие

$$
\nu_{n l} \equiv \lambda_{n}-\lambda_{l}+V>0 \quad \text { для всех } n \neq l .
$$

Для этих номеров существуют решения системы уравнений $(28),(29)$, которые при $\theta \rightarrow 0$ сходятся к (34). Асимптотика этих решений при $\theta \rightarrow 0$ имеет вид

$$
m_{n}^{(l)} \sim g \exp \left(-\frac{\nu_{n l}}{\theta}\right) \forall n \neq l, \quad 1-m_{l}^{(l)} \sim g \sum_{n \neq l} \exp \left(-\frac{\nu_{n l}}{\theta}\right) .
$$

Итак, в зависимости от спектра $\lambda_{n}, n \in \mathbb{Z}$, при достаточно малых значениях температуры $\theta$ система уравнений $(28),(29)$ имеет множество решений. Эти решения кроме точки глобального минимума содержат также точки локального минимума функции (26). Значения функции (26) в точках локального минимума равны свободной энергии метастабильных состояний. Рассмотрим функцию (26) при $\theta=0$. Она в этом случае совпадает с энергетическим спектром системы (21). Рассмотрим энергию системы в случае, когда почти все частицы находятся на энергетическом уровне $\lambda_{l}$, это означает, что вьполняются условия

$$
\tilde{N}_{n} \ll N \quad \forall n \neq l .
$$

Выражая $\tilde{N}_{l}$ из равенства $(23)$ и подставляя результат в $(21)$, получим в силу $(37)$, что энергетический спектр системы в этой области имеет вид

$$
\mathbf{E}(\tilde{N}) \approx \lambda_{l} N-\frac{V N}{2}+\sum_{n \neq l}\left(\lambda_{n}-\lambda_{l}+V\right) \tilde{N}_{n}
$$

Гамильтониану (18) соответствует уравнение Хартри и система уравнений в вариациях. Каждому $l \in \mathbb{Z}$ соответствует решение уравнения Хартри, описывающее состояние

$$
\widetilde{N}_{n}^{(l)}=N \delta_{n l}
$$

кроме того, собственные значения системы уравнений в вариациях, отвечающей этому решению уравнения Хартри, совпадают с

$$
\nu_{n l}=\lambda_{n}-\lambda_{l}+V, \quad n \neq l .
$$


Мы показали в [11], [12], что если собственные значения системы уравнений в вариациях для решения уравнения Хартри действительны и неотрицательны, то такое решение соответствует основному или метастабильному состоянию системы. Это означает, что всем $l$, для которых выполняется (35), при $\theta=0$ соответствуют метастабильные состояния системы бозонов. По аналогии со случаем нулевой температуры решениям системы уравнений $(28),(29)$ при $\theta \neq 0$ соответствует температурное метастабильное состояние, если точка (27) является точкой локального минимума. Заметим теперь, что при очень больших температурах у системы уравнений (28), (29) есть только одно решение, соответствующее глобальному минимуму функции (26). Асимптотика этого решения при $\theta \rightarrow \infty$ имеет вид

$$
n_{m}(\theta) \sim g \frac{e^{-\lambda_{m} / \theta}}{\sum_{l=-\infty}^{\infty} e^{-\lambda_{l} / \theta}} .
$$

Единственность решения при больших температурах означает, что все метастабильные состояния с увеличением температуры исчезают. Температура, при которой исчезает метастабильное состояние, является критической для этого состояния.

Рассмотрим вопрос о поведении энтропии и теплоемкости метастабильных состояний при приближении к критической температуре. Рассмотрим метастабильное состояние, которому соответствует решение системы уравнений $(28),(29)$, сходящееся при $\theta \rightarrow 0$ к (34) для некоторого $l$, для которого вьполняется (35). Будем считать далее, что функция $\varepsilon(p)$ такова, что $\varepsilon(0)<\varepsilon(p)$ при $p \neq 0$. Тогда согласно $(7) \lambda_{0}<\lambda_{l}$ при $l \neq 0$. Поэтому решение системы уравнений $(28),(29)$, сходящееся при $\theta \rightarrow 0$ к (34) для $l=0$, соответствует основному температурному состоянию системы $N$ бозонов. Кроме того, условие (35) становится эквивалентно условию $\lambda_{l}-\lambda_{0}<V$.

Будем считать, что для $l \neq 0$ вьполнено это неравенство. Условие того, что соответствуюшее решение $m_{n}^{(l)}(\theta)$ системы уравнений $(28),(29)$ определяет по формуле $(27)$ точку локального минимума функции (26) при условии (23), имеет вид системы неравенств:

$$
\begin{gathered}
\alpha_{n}^{(l)}(\theta) \equiv-V+\frac{\theta g}{m_{n}^{(l)}(\theta)\left(g+m_{n}^{(l)}(\theta)\right)}>0 \quad \forall n \neq l, \\
\alpha_{l}^{(l)}(\theta) \equiv-V+\frac{\theta g}{m_{l}^{(l)}(\theta)\left(g+m_{l}^{(l)}(\theta)\right)}<0, \quad-\sum_{n \neq l} \frac{\alpha_{l}^{(l)}(\theta)}{\alpha_{n}^{(l)}(\theta)}<1 .
\end{gathered}
$$

Заметим, что эти неравенства вьполняются для (36) при $\theta \rightarrow 0$. Неравенства (42) получаются из условия положительности второй вариации функции $(26)$, вариация берется при условии (23).

Метастабильное состояние исчезает при температуре, при которой последнее неравенство из (42) становится равенством. Будем обозначать эту критическую температуру $\theta_{c}^{(l)}$. Из $(42)$ и $(28),(29)$ при $\theta<\theta_{c}^{(l)}$ следует, что $m_{n}^{(l)}(\theta)$ является растущей функцией переменной $\theta$ при $n \neq l$, а $m_{l}^{(l)}(\theta)$ является убывающей функцией $\theta$, кроме того получается, что $m_{l}^{(l)}(\theta)>m_{n}^{(l)}(\theta)>m_{n^{\prime}}^{(l)}(\theta)$, если $\lambda_{n}<\lambda_{n^{\prime}}$ и $n, n^{\prime} \neq l$.

Из (30) следует выражение для удельной энтропии метастабильного состояния в пределе при $N \rightarrow \infty$ :

$$
s^{(l)}(\theta)=\sum_{n=-\infty}^{\infty}\left(\left(g+m_{n}^{(l)}\right) \ln \left(1+\frac{m_{n}^{(l)}}{g}\right)-m_{n}^{(l)} \ln \left(\frac{m_{n}^{(l)}}{g}\right)\right),
$$


где для краткости написания формулы у $m_{n}^{(l)}(\theta)$ опущен аргумент $\theta$. Дифференцируя (43), получим

$$
\frac{\partial s}{\partial \theta}=\sum_{n \neq l} \frac{\partial m_{n}^{(l)}}{\partial \theta} \ln \left(\frac{g+m_{n}^{(l)}}{m_{n}^{(l)}} \frac{m_{l}^{(l)}}{g+m_{l}^{(l)}}\right)>0 .
$$

Последнее неравенство следует в силу свойств $m_{n}^{(l)}(\theta)$. Поскольку при критической температуре последнее из неравенств (42) является равенством, можно показать, что при $\theta \rightarrow \theta_{c}^{(l)}-0$ решения уравнений $(28),(29)$ ведут себя следуюшим образом:

$$
m_{n}^{(l)}(\theta)-m_{n}^{(l)}\left(\theta_{c}^{(l)}\right) \approx \frac{C^{(l)}}{\alpha_{n}^{(l)}\left(\theta_{c}^{(l)}\right)} \sqrt{\theta_{c}^{(l)}-\theta} \quad \forall n \in \mathbb{Z},
$$

где число $C^{(l)}$ - отрицательное число. Подстановка (45) в (43) показьвает, что производная удельной энтропии по температуре - эта величина равна теплоемкости, деленной на температуру - при $\theta \rightarrow \theta_{c}^{(l)}-0$ стремится к бесконечности как $1 / \sqrt{\theta_{c}^{(l)}-\theta}$. Это значит, что проектирование лагранжева многообразия, соответствующего метастабильному состоянию, на ось $\theta$ становится неоднозначным в окрестности критической температуры [4], [13]. Производная температуры по энтропии при приближении к критической температуре обращается в нуль; поэтому, а также в силу (44), как это уже было отмечено выше, лагранжево многообразие однозначно проектируется на ось $s$. Отметим, что из свойств $m_{n}^{(l)}(\theta)$ следует, что при $\theta<\theta_{c}^{(l)}$ вьполняется

$$
\begin{array}{cc}
m_{0}^{(l)}(\theta)<m_{n}^{(l)}(\theta) & \forall n \neq 0, l, \\
\alpha_{0}^{(l)}(\theta)<\alpha_{n}^{(l)}(\theta) & \forall n \neq 0, l .
\end{array}
$$

Неравенства (46) означают, что потенциальньй барьер между энергетическим уровнем $\lambda_{l}$ и $\lambda_{0}$ меньше, чем энергетический барьер между $\lambda_{l}$ и $\lambda_{n}$ при $n \neq 0, l$. Это значит, что рассматриваемая система бозонов, находящаяся в метастабильном состоянии с номером $l$, при достижении критической температуры скачком изменяет свое состояние и переходит в основное температурное состояние. Это фазовьй переход нулевого рода, поскольку скачком изменяются не только энтропия и теплоемкость, но также и свободная энергия.

Когда величина $\min _{n \neq 0}\left|\lambda_{n}-\lambda_{0}\right|=\delta$ мала, разность свободных энергий при фазовом переходе нулевого рода из самого нижнего метастабильного состояния в основное состояние также является малой, а теплоемкость при таком переходе имеет особенность. Асимптотика статистической суммы около критической точки дается асимптотикой канонического оператора в окрестности фокальной точки. Эта асимптотика имеет вид функции типа Эйри от мнимого аргумента и имеет особенность при $N \rightarrow \infty$. Можно подобрать параметры $\delta \ll 1, N \gg 1$ и $L \gg 1$ так, что вид точки фазового перехода будет в точности совпадать с $\lambda$-точкой [14]-[24].

\section{3. Критическая температура и фазовый переход для слабо неидеального бозе-газа}

Рассмотрим уравнение Шрёдингера для системы $N$ бозонов в слабо неидеальном бозе-газе, следуя работе Боголюбова [8] в трехмерном торе со сторонами длины $L$. Пусть 
бозоны попарно взаимодействуют между собой, а $\varepsilon V(x-y)$ - потенциал этого взаимодействия, функция на торе, $\varepsilon=1 / N$. Вариационньй метод Боголюбова при температуpe $\theta$ приводит к следующему выражению для свободной энергии такой системы ${ }^{1}$ :

$$
F\left(\theta, k_{0}\right)=F\left(\theta, k_{0},\{N(\theta)\}\right),
$$

где $F(\theta,\{N\})$ - следующая функция:

$$
\begin{aligned}
& F\left(\theta, k_{0},\{N\}\right)=\frac{\hbar^{2} k_{0}^{2}}{2 m} N+\frac{\varepsilon}{2 L^{3}} \tilde{V}(0) N(N-1)+\sum_{k \neq k_{0}}\left(\frac{\hbar^{2} k^{2}}{2 m}-\frac{\hbar^{2} k_{0}^{2}}{2 m}\right) N_{k} \\
& +\frac{\varepsilon}{L^{3}} \sum_{k \neq k_{0}}\left(\tilde{V}(0)+\widetilde{V}\left(k-k_{0}\right)\right)\left(N N_{k}-2 N_{k}^{2}-\sum_{k^{\prime} \neq k_{0}} N_{k} N_{k^{\prime}}\right) \\
& +\frac{\varepsilon}{2 L^{3}} \sum_{k \neq k^{\prime} \neq k_{0}}\left(\widetilde{V}(0)+\widetilde{V}\left(k-k^{\prime}\right)\right) N_{k} N_{k^{\prime}} \\
& +\frac{\varepsilon}{L^{3}} \sum_{k \neq k_{0}} \tilde{V}(0) N_{k}^{2}+\frac{\varepsilon}{2 L^{3}} \tilde{V}(0)\left((1-2 N) \sum_{k \neq k_{0}} N_{k}+\sum_{k \neq k^{\prime} \neq k_{0}} N_{k} N_{k^{\prime}}+2 \sum_{k \neq k_{0}} N_{k}^{2}\right) \\
& +\theta \sum_{k \neq k_{0}}\left(N_{k} \ln \left(N_{k}\right)-\left(N_{k}+1\right) \ln \left(N_{k}+1\right)\right),
\end{aligned}
$$

где $m$ - масса бозонов, $\hbar$ - постоянная Планка, $k, k^{\prime}$ - произвольные трехмерные векторы вида $2 \pi / L\left(n_{1}, n_{2}, n_{3}\right), n_{1}, n_{2}, n_{3}$ - целые числа, $k_{0}$ - некоторый выделенньй вектор такого вида, а $\widetilde{V}$ выражается формулой

$$
\widetilde{V}(k)=\int d x V(x) e^{-i k x},
$$

наконец $\{N\}$ обозначает набор переменных $N_{k}, k \neq k_{0}$, a $\{N(\theta)\}$ - точка минимума функции (48). Заметим, что функция (48) соответствует случаю, когда в системе бозонов есть конденсат, состояший из частиц с импульсом $\hbar k_{0}$. Пусть $k_{0} \neq 0$ меньше скорости Ландау, т.е. для него выполнено условие

$$
\min _{k \neq k_{0}}\left(\frac{\hbar^{2} k^{2}}{2 m}-\frac{\hbar^{2} k_{0}^{2}}{2 m}+\frac{\tilde{V}\left(k-k_{0}\right)}{L^{3}}\right)>0 .
$$

Такому $k_{0}$ соответствует метастабильное состояние. Уравнения для определения точки минимума функции (48)

$$
\frac{\partial F(\theta,\{N\})}{\partial N_{k}}=0 \quad \forall k \neq k_{0},
$$

эквивалентны системе температурных уравнений Хартри. В силу (50) уравнения (51) имеют решения при достаточно малых температурах $\theta$, асимптотика этих решений при $\theta \rightarrow 0$ имеет вид

$$
N_{k} \approx e^{-\omega_{k k_{0}} / \theta} \quad \forall k \neq k_{0}
$$

\footnotetext{
${ }^{1} \mathrm{~K}$ этим же уравнениям приводит асимптотика ультравторично квантованных уравнений термодинамики [10].
} 
где

$$
\omega_{k k_{0}}=\frac{\hbar^{2} k^{2}}{2 m}-\frac{\hbar^{2} k_{0}^{2}}{2 m}+\frac{\widetilde{V}\left(k-k_{0}\right)}{L^{3}} .
$$

Заметим, что подстановка (52) в (48) приводит к выражению отличному от свободной энергии, получаемой суммированием по квазичастищам. Пусть теперь $k_{0}$ меньше скорости Ландау, но очень к ней близко. Это значит, что минимум в (50) достигается при $k=0$ и этот минимум стремится к 0 при $N \rightarrow \infty$. Для определенности будем считать, что

$$
\omega_{0 k_{0}}=\frac{\alpha}{N^{\sigma}}
$$

где $1 / 2<\sigma$. Поскольку $\omega_{k k_{0}}$ при $k \neq 0$ есть величина порядка 1 , из (54) следует, что для любой температуры справедливо

$$
N_{k} \ll N_{0}
$$

В силу (55) в пределе при $N \rightarrow \infty$ главньй вклад в функцию (48) дают слагаемые, содержащие $N_{0}$, а слагаемые, не содержащие $N_{0}$, можно не учитывать. Система уравнений (51) для определения точки минимума в таком случае переходит в уравнение

$$
\frac{\alpha}{N^{\sigma}}+\frac{\beta}{N} N_{0}-\frac{\theta}{N_{0}} \approx 0
$$

где

$$
\beta=2\left(2 \widetilde{V}\left(k_{0}\right)+\widetilde{V}(0)\right)>0 .
$$

Уравнение (56) тривиально приводится к квадратному уравнению. Исследование этого уравнения показьвает, что оно имеет два решения при $0<\theta<\theta_{c}$, где

$$
\theta_{c}=\frac{\alpha^{2}}{4 \beta N^{2 \sigma-1}}
$$

а при $\theta_{c}<\theta$ уравнение (56) решений не имеет. При $\theta \rightarrow \theta_{c}-0$ решения уравнения (56) имеют вид

$$
N_{0}(\theta) \approx \sqrt{\frac{N \theta_{c}}{\beta}}\left(1-\sqrt{1-\frac{\theta}{\theta_{c}}}\right) .
$$

Подстановка (59) в (48) приводит к свободной энергии, вторая производная которой обращается в бесконечность при $\theta \rightarrow \theta_{c}-0$. Это означает, что теплоемкость метастабильного состояния сильно растет около критической для этого метастабильного состояния температуры:

$$
C\left(\theta, k_{0}\right)=-\theta \frac{\partial^{2} F\left(\theta, k_{0}\right)}{\partial \theta^{2}} \approx \frac{1}{2 \sqrt{\theta_{c}\left(\theta_{c}-\theta\right)}} .
$$

Таким образом, оказьвается, что в модели Боголюбова свойства метастабильных состояний аналогичны свойствам метастабильных состояний в двухуровневой модели. А именно, метастабильные состояния исчезают при критической температуре, а теплоемкость этих состояний при приближении к критической температуре растет по закону $1 / \sqrt{\theta_{c}-\theta}$.

Следовательно, для слабо неидеального бозе-газа в модели Боголюбова возникает та же картина фазового перехода нулевого рода и эффект фонтанирования.

В заключение выражаю глубокую благодарность Г. В. Ковалю за помощь. 


\section{СПИСОК ЦИТИРОВАННОЙ ЛИТЕРАТУРЫ}

[1] Кубо Р. Термодинамика. М.: Мир, 1970.

[2] Ландау Л. Л., Лифшиц Е. М. Квантовая механика. М.: Гостехиздат, 1948.

[3] Маслов В.П. Математические аспекты теории слабо неидеального бозе и ферми газа на кристаллической подложке // Функцион. анализ и его прилож. 2003. Т. 37. № 2. С. 16-27.

[4] Маслов В. П. Геометрическое "квантование" термодинамики и статистические поправки в критических точках // ТМФ. 1994. Т. 101. № 3. С. 433-441.

[5] Маслов В. П. Аксиомы нелинейного осреднения в финансовой математике и динамика курса акций // Теория вероятн. и ее применения. 2003. Т. 48. № 4. С. 799-810.

[6] Боголюбов Н. Н. Избранные труды в трех томах. Т. 1, 2. Киев: Наукова думка, 1970.

[7] Maslov V.P., Molchanov S. A., Gordon A. Ya. Behaviour of generalized eigenfunctions at infinity and Schrödinger conjecture // Russ. J. Math. Phys. 1993. V. 1. № 1. P. 71-104.

[8] Боголюбов Н. Н. К теории сверхтекучести // Изв. АН СССР. Сер. физич. 1947. Т. 11. № 1. C. $77-90$.

[9] Маслов В.П. Аксиомы нелинейного осреднения в финансовой математике и аналог фазового перехода // Докл. РАН. 2003. Т. 393. №6. С. 735-739.

[10] Маслов В.П. Квантование термодинамики и ультравторичное квантование. М.: Институт компьютерных исследований, 2001.

[11] Маслов В. П. Новое представление оператора Гамильтона для бозонов и фермионов. Квантование свободной энергии и зависимость критерия Ландау от температуры // Матем. заметки. 2000. Т. 68. №6. С. 945-947.

[12] Маслов В. П., Шведов О. Ю. Метод комплексного ростка в задаче многих частиц и квантовой теории поля. М.: Эдиториал УРСС, 2000.

[13] Маслов В.П. Аналитическое продолжение асимптотических формул и аксиоматика термодинамики и квазитермодинамики // Функцион. анализ и его прилож. 1994. Т. 28. № 4. C. $28-41$.

[14] Маслов В.П. Модель слабо неидеалного бозе-газа. Фазовый переход в сверхтекучем состоянии и эффект фонтанирования // Вестн. МГУ. Сер. физ., астрономия. 2003. №1. С. 3-5.

[15] Маслов В. П. Двухуровневая модель слабо неидеального бозе-газа. Фазовый переход в метастабильном (сверхтекучем) состоянии // Докл. РАН. 2003. Т. 389. № 4. С. 468-469.

[16] Маслов В. П. Об одной модели слабонеидеального бозе-газа, приводящей к эффекту фонтанирования // ТМФ. 2003. Т. 135. № 3. С. 524-528.

[17] Маслов В. П. Фазовые переходы бозе-газа в двухзонной модели кристалла. Термомеханический эффект // УМН. 2003. Т. 58. №2 2(350). С. 157-158.

[18] Маслов В. П. Об одной модели высокотемпературной сверхпроводимости. // Докл. РАН. 2003. Т. 391. № 5. С. $605-609$.

[19] Маслов В.П. О модели высокотемпературной сверхпроводимости // Матем. заметки. 2003. T. 73. №6. C. 942-946.

[20] Maslov V.P. A new exactly solvable model of high-temperature superconductivity // Russ. J. Math. Phys.. V. 11. № 2. P. 199-208.

[21] Маслов В. П. О точно решаемой модели низкотемпературной сверхпроводимости // Докл. PAH. 2003. T. 397. №6. C. 1-3.

[22] Маслов В.П. Точная модель высокотемпературной сверхпроводимости // Докл. РАН. 2004. T. 398. № 3 .

[23] Маслов В. П. Учет отталкивания для модели высокотемпературной проводимости и сверхтекучести бозе-газа на кристаллической подложке. Фазовый переход нулевого рода // Докл. PAH. 2004. T. 398. №6.

[24] Маслов В.П. Об одной точно решаемой модели сверхтекучести // Докл. РАН. 2004. T. 399. № 1.

Московский государственный университет им. М.В.Ломоносова 